\title{
SEMIGROUP STRUCTURES FOR FAMILIES OF FUNCTIONS, III
}

\author{
$\mathfrak{R}_{T^{*} \text {-SEMIGROUPS }}$ \\ KENNETH D. MAGILL, Jr.
}

(Received 22 August 1966)

\section{Introduction}

Let $\mathscr{F}_{X}$ and $\mathscr{F}_{Y}$ denote families of subsets of the nonempty sets $X$ and $Y$ respectively and let $\mathfrak{f}$ be a function mapping $Y$ onto $X$ with the property that $\mathfrak{f}[H] \in \mathscr{F}_{X}$ for each $H \in \mathscr{F}_{Y}$. Then the family $\mathscr{S}$ of all functions $f$ mapping $X$ into $Y$ such that $f[H] \in \mathscr{F}_{Y}$ for each $H \in \mathscr{F}_{X}$ is a semigroup if the product $f g$ of two such functions $f$ and $g$ is defined by $f g=f \circ f \circ g$ (i.e., $(f g)(x)=f(f(g(x)))$ for each $x$ in $X)$. With some restrictions on the families $\mathscr{F}_{X}$ and $\mathscr{F}_{X}$ and the function $\mathfrak{f}$, these are the semigroups mentioned in the title and are the objects of investigation in this paper. The restrictions on $\mathscr{F}_{X}, \mathscr{F}_{Y}$ and $f$ are sufficiently mild so that the semigroups considered here include such semigroups of functions on topological spaces as semigroups of closed functions, semigroups of connected functions, etc.

Before continuing, it will be convenient to define some terms.

Definition (1.1). For a nonempty set $X$, we use the symbol $\mathscr{P}(X)$ to denote the family of all subsets of $X$. The subfamily of $\mathscr{P}(X)$ which consists of $X$ and all singletons of $X$ is referred to as the core of $\mathscr{P}(X)$.

Definition (1.2). Let $\mathscr{F}$ be any subfamily of $\mathscr{P}(X)$. A function $f$ mapping $X$ into $X$ which has the property that $f[H] \in \mathscr{F}$ for each $H \in \mathscr{F}$ is referred to as an $\mathscr{F}$-invariant function.

Definition (1.3). A subfamily $\mathscr{F}$ of $\mathscr{P}(X)$ is referred to as an $\Re$-family if it contains the core of $\mathscr{P}(X)$ and for each set $H$ in $\mathscr{F}$, there exists an $\mathscr{F}$-invariant function $f$ such that $f[X]=H$.

Definition (1.4). An $\Re$-space is a pair $(X, \mathscr{F})$ where $X$ is a nonempty set and $\mathscr{F}$ is an $\Re$-family of subsets of $X$.

We will often take the liberty (as one does in referring to a topological space) of referring to $X$ itself as an $\Re$-space. It is to be understood that some $\Re$-family of subsets is associated with $X$. $\Re$-families are investigated 
in Section 2. The results obtained there show that these families are rather abundant. For example, the family of all closed subsets of a $T_{1}$ topological space is an $\Re$-family and the family of all connected subsets of a connected completely regular Hausdorff space with cardinality $c$ (the cardinality of the continuum) is an $\Re$-family.

Definition (1.5). Let $\left(X, \mathscr{F}_{X}\right)$ and $\left(Y, \mathscr{F}_{Y}\right)$ be two $\Re$-spaces. A function $f$ from $X$ into $Y$ is an $\Re$-function if $f[H] \in \mathscr{F}_{Y}$ for each $H \in \mathscr{F}_{X}$.

Definition (1.6). A function $f$ from $X$ onto $Y$ is referred to as an $\Re$ bijection if $f$ is a bijection from $X$ onto $Y$ and both $f$ and $f^{\leftarrow}$ are $\Re$-functions.

Now let $X$ and $Y$ be $\Re$-spaces and suppose that for every pair of distinct points $p$ and $q$ of $X$, there exists an $\Re$-function $f$ mapping $X$ into $Y$ with the property that $f(p) \neq f(q)$. We note that since $\mathscr{F}_{Y}$ (the $\Re$-family associated with $Y$ ) contains all singletons of $Y$, all the constant functions mapping $X$ into $Y$ are $\Re$-functions. Therefore, if $\mathfrak{f}$ is any $\Re$-function which maps $Y$ onto $X$, the family of all $\Re$-functions from $X$ into $Y$ is an $\mathfrak{S}^{*}$-semigroup [8] if we define the product $f g$ of two such functions by $f g=f \circ f \circ g$. This particular $\mathfrak{S}^{*}$-semigroup will be denoted by $\Re_{T}^{*}(X, Y, \mathfrak{f})$ and will be referred to as an $\Re_{T^{*}}^{*}$-semigroup. It follows from Theorem (2.7) of [8] that if $\varphi$ is any isomorphism from the $\Re_{T}^{*}$-semigroup $\Re_{T}^{*}(X, Y, \mathfrak{f})$ onto the $\Re_{T}^{*}$-semigroup $\mathfrak{R}_{T}^{*}(U, V, \mathfrak{g})$, then there exist unique bijections $\mathfrak{h}$ and $t$ from $X$ onto $U$ and $Y$ onto $V$ respectively such that for each $f$ in $\Re_{T}^{*}(X, Y, \mathfrak{f})$, the following diagram commutes.

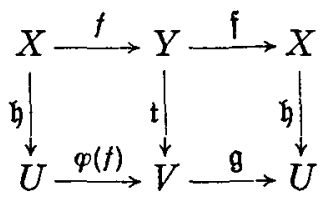

Section 3 is devoted to finding additional conditions which will insure that the bijections $\mathfrak{h}$ and $\mathfrak{t}$ are $\Re$-bijections. Results obtained in Sections $\mathbf{2}$ and $\mathbf{3}$ generalize Theorem (2.10) of [7] which states that if $\Gamma(X)$ and $\Gamma(Y)$ denote respectively the semigroups of closed functions from the $T_{1}$ topological space $X$ into itself and the $T_{1}$ topological space $Y$ into itself, then a mapping $\varphi$ from $\Gamma(X)$ onto $\Gamma(Y)$ is an isomorphism if and only if there exists a homeomorphism $\mathfrak{h}$ from $X$ onto $Y$ such that $\varphi(f)=\mathfrak{h} \circ f \circ \mathfrak{h}^{+}$for each $f$ in $\Gamma(X)$. Generalizations are also obtained of certain results in [7] which involve semigroups of connected functions.

Automorphisms of certain $\mathfrak{R}_{\boldsymbol{T}}^{*}$-semigroups are studied in Section 4 . The first result of that section gives a number of conditions which are equivalent to the condition that certain $\mathfrak{R}_{T}^{*}$-semigroups have inner automorphisms. Another result of that section determines the automorphism groups for 


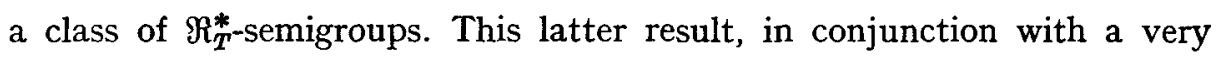
interesting result of $\mathrm{J}$. de Groot enables us to answer in the affirmative a question of the type considered by him in [3]. Generally stated, the question is, "For a given class $\mathscr{C}$ of algebraic systems, is every group isomorphic to the automorphism group of some member of $\mathscr{C}$ ?" De Groot gives an affirmative answer in [3] for the class of all rings. Furthermore, he points out that Birkhoff [1] has given an affirmative answer for the class of distributive lattices while a result of his shows that the answer is negative for the class of complemented distributive lattices. He also points out that the answer is negative for the class of all groups. In particular, no group has an automorphism group which is cyclic of odd order. However, we are able to give an affirmative answer for the class of all semigroups. The last result of the paper states that every group is isomorphic to the automorphism group of some semigroup.

\section{Some results on $\mathfrak{A}$-families}

The results of this section show that $\Re$-families are rather abundant and that many familiar families of sets are $\Re$-families.

THEOREM (2.1). Let $\mathscr{F}$ be any family of mutually disjoint subsets of a nonempty set $X$. Then the family $\mathscr{F} *$ consisting of $\mathscr{F}$ and the sets in the core of $\mathscr{P}(X)$ is an $\Re$-family.

Proof. Let $H$ be a nonempty set belonging to $\mathscr{F} *$. Choose $p$ in $H$ and define a function $f$ mapping $X$ onto $H$ by

$$
\begin{aligned}
& f(x)=x \text { for } x \text { in } H \text { and } \\
& f(x)=p \text { for } x \text { in } X-H .
\end{aligned}
$$

It follows that $f$ is an $\mathscr{F} *$-invariant function and hence that $\mathscr{F} *$ is an $\Re$ family.

THEOREM (2.2). Let $X$ be infinite and suppose $\mathscr{F}$ consists of the sets in the core of $\mathscr{P}(X)$ and a finite number of infinite subsets of $X$. Then $\mathscr{F}$ is an $\Re$-family.

Proof. We first prove by induction that if $H$ is any infinite subset of $X$ and $K_{1}, K_{2}, \cdots, K_{n}$ are $n$ subsets of $X$, one of which is $X$ itself, then there exists a positive integer $m$ such that $1 \leqq m \leqq n$ and

$$
\operatorname{card}\left(\left(H \cap K_{i_{1}} \cap K_{i_{2}} \cap \cdots \cap K_{i_{m}}\right)-\left(K_{i_{m+1}} \cup \cdots \cup K_{i_{n}}\right)\right)=\operatorname{card}(H)
$$

where, for any set $Y$, card $(Y)$ denotes the cardinal number of the set $Y$. Suppose $n=1$. Then $K_{1}=X$ and

$$
\operatorname{card}\left(H \cap K_{1}\right)=\operatorname{card}(H) \text {. }
$$


Thus, in this case, $m=n=1$. Now suppose (2.2.1) holds for $n=r$ and let $K_{1}, K_{2}, \cdots, K_{r}, K_{r+1}$ be $r+1$ infinite subsets of $X$ where $K_{i}=X$ for some $i<r+1$. Let

$$
A=\left(H \cap K_{i_{1}} \cap \cdots \cap K_{i_{m}}\right)-\left(K_{i_{m+1}} \cup \cdots \cup K_{i_{r}}\right) .
$$

Since $\left(A \cap K_{r+1}\right) \cup\left(A-K_{r+1}\right)=A$, we must have either

$$
\text { card }\left(A \cap K_{r+1}\right)=A \text { or }
$$$$
\text { card }\left(A-K_{r+1}\right)=A \text {. }
$$

Now

and

$$
A \cap K_{r+1}=\left(H \cap K_{i_{1}} \cap \cdots \cap K_{i_{m}} \cap K_{r+1}\right)-\left(K_{i_{m+1}} \cup \cdots \cup K_{i_{r}}\right)
$$

$$
A-K_{r+1}=\left(H \cap K_{i_{1}} \cap \cdots \cap K_{i_{m}}\right)-\left(K_{i_{m+1}} \cup \cdots \cup K_{i_{r}} \cup K_{r+1}\right) .
$$

Therefore, since card $(A)=$ card $(H)$, it follows that (2.2.1) holds for $n=k+1$ and hence for each positive integer $n$.

Now let $H$ be any infinite set in $\mathscr{F}$ which is different from $X$ and denote the remaining infinite sets in $\mathscr{F}$ by $K_{1}, K_{2}, \cdots, K_{n}$. It follows from (2.2.1) that there exists a bijection $h$ from $\left(H \cap K_{i_{1}} \cap \cdots \cap K_{i_{m}}\right)-\left(K_{i_{m+1}} \cup \cdots \cup K_{i_{n}}\right)$ (which we will denote by $B$ ) onto $H$. Choose a point $p$ in $H^{+1}$ and define a mapping $f$ from $X$ onto $H$ by

$$
\begin{aligned}
& f(x)=h(x) \text { for } x \text { in } B \text { and } \\
& f(x)=p \text { for } x \text { in } X-B .
\end{aligned}
$$

Note that $f[V]=H$ for $V=H, \quad K_{i_{1}}, \cdots, K_{i_{m}}$ and $f[V]=\{p\}$ for $V=K_{i_{m+1}}, K_{i_{m+2}}, \cdots, K_{i_{n}}$. It follows that $f$ is an $\mathscr{F}$-invariant function. To complete the proof, we must show that $X$ and the singletons of $X$ are all ranges of $\mathscr{F}$-invariant functions. But this is a consequence of the fact that the identity mapping and the constant mappings are all $\mathscr{F}$-invariant functions.

The following example shows that the conclusion of Theorem (2.2) does not hold for an infinite number of infinite subsets of $X$.

Example (2.3). Let $X$ be an infinite set and $A$ a subset of $X$ with the properties

$$
X-A \text { is infinite and }
$$

$$
\text { card }(X-A)<\operatorname{card} A \text {. }
$$

For any ordinal number $\alpha$, we let $L(\alpha)=\{\beta: \beta$ is an ordinal number and $\beta<\alpha$. Let $\alpha$ be the first ordinal number for which card $(L(\alpha))=$ card $(A)$. Thus, if $\beta<\alpha$, card $(L(\beta))<\operatorname{card}(A)$. Now there exists a bijection $h$ 
from $L(\alpha)$ onto $A$ and for each ordinal number $\beta<\alpha$, we define a subset $H_{\beta}$ by

$$
H_{\beta}=(X-A) \cup h[L(\beta)] .
$$

Conditions (2.3.1) and (2.3.2) imply that card $(A)$ is infinite. This, together with the fact that $\alpha$ is the first ordinal for which card $(L(\alpha))=$ card $(A)$, implies that $\alpha$ is a limit ordinal, for suppose $\alpha$ has an immediate predecessor $\beta$. Then $L(\alpha)=L(\beta) \cup\{\beta\}$ and it would follow that card $(A)=\operatorname{card}(L(\alpha))=$ card $(L(\beta))$ which would contradict the choice of $\alpha$ as the first ordinal for which card $(A)=$ card $(L(\alpha))$. Since $\alpha$ is a limit ordinal, it follows that

$$
X=\cup\left\{H_{\beta}: \beta<\alpha\right\}
$$

and that for any $\beta<\alpha$,

$$
\text { card }\left(H_{\beta}\right)=\operatorname{card}(X-A)+\operatorname{card}(L(\beta)) .
$$

But since card $(X-A)<$ card $(A)$ and card $(L(\beta))<$ card $(A)$, it follows that

$$
\text { card }\left(H_{\beta}\right)<\operatorname{card}(A) \text {. }
$$

Now let $\mathscr{F}$ consist of the sets in the core of $\mathscr{P}(X)$ along with $A$ and all the sets $H_{\beta}(\beta<\alpha)$ and let $f$ be any $\mathscr{F}$-invariant function mapping $X$ into $A$. Let us consider the set $H_{0}$. By (2.3.4), card $\left(H_{0}\right)<$ card $(A)$. This, together with the fact that the only proper subsets of $A$ which belong to $\mathscr{F}$ are singletons, implies that $f\left[H_{0}\right]$ is a singleton, i.e., $f\left[H_{0}\right]=\{p\}$ for some $p$ in $A$. Now let $\beta$ be any other ordinal number less than $\alpha$. The same argument allows us to conclude that $f\left[H_{\beta}\right]$ is a singleton. In fact, since $H_{\beta} \cap H_{0} \neq \emptyset$, it follows that $f\left[H_{\beta}\right]=\{p\}$. From (2.3.3), we see that

$$
f[X]=f\left[\cup\left\{H_{\beta}: \beta<\alpha\right\}\right]=\cup\left\{f\left[H_{\beta}\right]: \beta<\alpha\right\}=\{p\} .
$$

But (2.3.1) and (2.3.2) imply $A$ is infinite. Thus $f[X] \neq A$ and we conclude that $A$ is not the range of any $\mathscr{F}$-invariant function. Thus $\mathscr{F}$ is not an $\mathfrak{R}$ family.

It follows immediately from Theorem (2.1) that if $\mathscr{F}$ is any subfamily of $\mathscr{P}(X)$ which consists of the core along with one other subset of $X$, then $\mathscr{F}$ is an $\Re$-family. However, the following example shows that there do exist families, consisting of the core and two other subsets, which are not $\Re$-families.

Example (2.4). Let $X$ be a finite set and let $A$ and $B$ be any two subsets of $X$ satisfying the following properties:

$$
B \cap(X-A) \neq \emptyset \text {, }
$$

$$
\text { card }(A \cap B)>1 \text {, }
$$




$$
\begin{aligned}
& \operatorname{card}(B)<\operatorname{card}(A), \\
& \operatorname{card}(X-(A \cup B))+1<\operatorname{card}(A) .
\end{aligned}
$$

Let $\mathscr{F}$ consist of the core of $\mathscr{P}(X)$ together with the sets $A$ and $B$. Now let $f$ be any $\mathscr{F}$-invariant mapping from $X$ into $A$. Since card $(B)<\operatorname{card}(A)$, $f[B] \neq A$. Thus $f[B]=\{p\}$ for some $p$ in $A$. Since card $(A \cap B)>1, f$ maps at least two points of $A$ onto $p$. This implies $f[A] \neq A$ and it follows that $f[A]=\{p\}$. Therefore $f[A \cup B]=\{p\}$. Now

$$
\begin{aligned}
\operatorname{card}(f[X]) & =\operatorname{card}(f[X-(A \cup B)] \cup f[A \cup B]) \\
& \leqq \operatorname{card}(f[X-(A \cup B)])+\operatorname{card}(f[A \cup B]) \\
& \leqq \operatorname{card}(X-(A \cup B))+1<\operatorname{card}(A) .
\end{aligned}
$$

Thus $f[X] \neq A$ and we conclude that $A$ is not the range of an $\mathscr{F}$-invariant function. Thus $\mathscr{F}$ is not an $\Re$-family. For an example of sets $X, A$, and $B$ satisfying conditions (2.4.1)-(2.4.4), let $X=\{1,2,3,4,5\}, A=\{1,2,3,4\}$ and $B=\{1,2,5\}$.

THEOREM (2.5). Let $X$ be any nonempty set and let $\mathscr{F}$ be a family of subsets of $X$ satisfying the following conditons:

(2.5.1) $\mathscr{F}$ contains the core of $\mathscr{P}(X)$,

(2.5.2) $\mathscr{F}$ is closed under finite intersections,

(2.5.3) if $H \in \mathscr{F}$ and $\{p\}$ is any singleton of $X$, then $H \cup\{p\} \in \mathscr{F}$.

Then $\mathscr{F}$ is an $\Re$-family.

Proof. Let $H$ be any nonempty set in $\mathscr{F}$ and choose $p$ in $H$. Define a function $f$ mapping $X$ onto $H$ by

$$
\begin{aligned}
& f(x)=p \text { for } x \text { in } X-H, \\
& f(x)=x \text { for } x \text { in } H .
\end{aligned}
$$

Then for any $F$ in $\mathscr{F}$,

$$
\begin{aligned}
f[F] & =f[(F \cap H) \cup(F \cap(X-H))] \\
& =f[F \cap H] \cup f[F \cap(X-H)]=(F \cap H) \cup f[F \cap(X-H)] .
\end{aligned}
$$

Now, $F \cap H \in \mathscr{F}$ by (2.5.2) and $f[F \cap(X-H)]$ is either $\emptyset$ or $\{p\}$. In either event, $(F \cap H) \cup f[F \cap(X-H)]$ belongs to $\mathscr{F}$ and we see that $H$ is the range of an $\mathscr{F}$-invariant function. Thus $\mathscr{F}$ is an $\Re$-family.

The following two corollaries are immediate consequences of Theorem (2.5).

COROllary (2.6). The family of all closed subsets of a $T_{1}$ topological space is an $\Re$-family. 
Corollary (2.7). Let $X$ be a Hausdorff space and let $\mathscr{F}$ denote the family of all compact subsets of $X$ together with $X$ itself. Then $\mathscr{F}$ is an $\Re$-family.

The following result is a reformulation of Theorem (3.2) of [7]. The proof carries over intact.

THEOREM (2.8). The family of all connected subsets of a connected completely regular Hausdorff space with cardinality $c$ (the cardinality of the continuum) is an $\Re$-family.

Proof. Let $K$ be any connected subset of $X$ and choose two distinct points $p$ and $q$ of $X$. Since $X$ is completely regular and Hausdorff, there exists a continuous function $f$ from $X$ into the closed unit interval $I$ with the properties $f(p)=0$ and $f(q)=1$. Since $f[X]$ is connected and contains both 0 and 1 , it follows that $f[X]=I$.

Now for any point $x$ in $I$ which is different from zero, let

$$
\text { - } a_{1} a_{2} a_{3} \cdots a_{n} \cdots \quad\left(a_{i}=0 \text { or } 1\right)
$$

denote the nonterminating binary expansion of $x$. Define a function $g$ from $I$ into $I$ by

$$
\begin{aligned}
& g(x)=\lim \sup \left(a_{1}+a_{2}+\cdots+a_{n}\right) / n \\
& g(0)=0 .
\end{aligned}
$$

The function $g$ is discussed in [5] on page 82. It has the interesting property that the image of any nondegenerate subinterval (open, closed or halfopen) of $I$ is $I$ itself. Since the cardinality of $K$ does not exceed that of $I$, there exists a function $h$ mapping $I$ onto $K$. It follows that $(h \circ g \circ f)[X]=K$. Moreover, if $H$ is any connected subset of $X$, then $f[H]$ is either a point of $I$ or a nondegenerate subinterval of $I$. In the former case, $(h \circ g \circ f)[H]$ is a point of $X$ and in the latter, $(h \circ g \circ f)[H]=K$. This completes the proof.

\section{Isomorphisms of $\mathfrak{R}_{T}^{*}$-semigroups}

Definition (3.1). Let $\left(X, \mathscr{F}_{X}\right)$ and $\left(Y, \mathscr{F}_{Y}\right)$ be two $\Re$-spaces and $\mathfrak{f}$ an $\Re$-function from $Y$ onto $X$. The triple $(X, Y, \mathfrak{f})$ is said to be $\Re$-admissible if the following conditions are satisfied:

(3.1.1) There exists an $\Re$-function from $X$ onto $Y$.

(3.1.2) If $p$ and $q$ are distinct points of $X$, then $f(p) \neq f(q)$ for some $\Re$ function $f$ mapping $X$ into $Y$.

$$
\mathfrak{f}^{-}[H] \in \mathscr{F}_{\boldsymbol{Y}} \text { for each } H \in \mathscr{F}_{\boldsymbol{X}} \text {. }
$$

Before proving the main result of this section, we recall two definitions and a result from [8] which are needed here. Let $\mathscr{S}$ be a family of functions with domains contained in a set $X$ and ranges contained in a set $Y$ and 
let $\mathfrak{f}$ be a function with domain equal to $Y$ and range contained in $X$ with the property that $f \circ f \circ g \in \mathscr{S}$ for each pair of elements $f$ and $g$ in $\mathscr{S}$.

Definition (3.2). $\mathscr{S}$ is referred to as an $\widetilde{S}$-semigroup and is denoted by $\mathfrak{S}(X, Y, \mathfrak{f})$ if the following two conditions are satisfied.

(3.2.1) For each pair $x_{1}$ and $x_{2}$ of distinct points of $X$, there exists a function $f$ in $\mathscr{S}$ whose domain contains both $x_{1}$ and $x_{2}$ with the property that $f\left(x_{1}\right) \neq f\left(x_{2}\right)$.

(3.2.2) For each $x$ in $X$ and $y$ in $Y$, there is a subset $A$ of $X$ containing $x$ such that $A_{y} \in \mathscr{S}\left(A_{y}\right.$ is the function whose domain is $A$ and which is defined by $A_{y}(p)=y$ for all $p$ in $A$ ).

Definition (3.3). An $\mathfrak{S}$-semigroup $\mathfrak{S}(X, Y, \mathfrak{f})$ is referred to as an $\mathfrak{S}^{*}$-semigroup and is denoted by $\mathfrak{S}^{*}(X, Y, \mathfrak{f})$ if $\mathfrak{f}$ is a surjection onto $X$.

Theorem (3.4). A bijection $\varphi$ from an $\mathfrak{S}^{*}$-semigroup $\mathfrak{S}^{*}(X, Y, \mathfrak{f})$ onto an $\mathfrak{S}^{*}$-semigroup $\mathfrak{S}^{*}(U, V, \mathfrak{g})$ is an isomorphism if and only if there exist bijections $\mathfrak{h}$ and $\mathrm{t}$ from $X$ onto $U$ and $Y$ onto $V$ respectively such that for each $f$ in $\mathfrak{S}^{*}(X, Y, \mathfrak{f}), \mathfrak{h}$ maps the domain, $\mathscr{D}(f)$, of $f$ bijectively onto the domain, $\mathscr{D}(\varphi(f))$, of $\varphi(f)$ and the following diagram commutes.

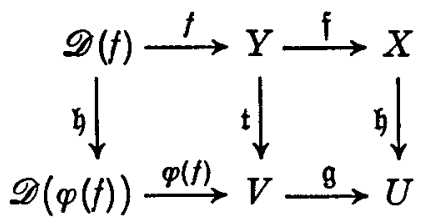

Moreover, the functions $\mathfrak{h}$ and $\mathfrak{t}$ are unique in the sense that if $\mathfrak{h}^{*}$ and $\mathfrak{t}^{*}$ are two mappings from $X$ into $U$ and $Y$ into $V$ respectively with the property that the resulting diagram commutes when $\mathfrak{h}$ is replaced by $\mathfrak{h}^{*}$ and $\mathrm{t}$ by $\mathrm{t}^{*}$, then $\mathfrak{h}=\mathfrak{h} *$ and $\mathfrak{t}=\mathfrak{t}^{*}$.

Now we are in a position to prove the main result of this section.

THEOREM (3.5). Let $(X, Y, \mathfrak{f})$ and $(U, V, \mathfrak{g})$ be $\Re$-admissible triples. Then a bijection $\varphi$ from $\Re_{T}^{*}(X, Y, \mathfrak{f})$ onto $\mathfrak{\Re}_{T}^{*}(U, V, \mathfrak{g})$ is an isomorphism if and only if there exist $\Re$-bijections $\mathfrak{h}$ and $\mathrm{t}$ from $X$ onto $U$ and $Y$ onto $V$ respectively such that the following diagram commutes.

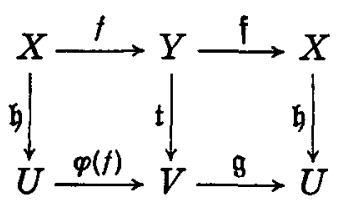

Moreover, the functions $\mathfrak{h}$ and $\mathrm{t}$ are unique in the sense that if $\mathfrak{h}^{*}$ and $\mathrm{t}^{*}$ are two 
mappings from $X$ into $U$ and $Y$ into $V$ respectively with the property that the resulting diagram commutes when $\mathfrak{h}$ is replaced by $\mathfrak{h}^{*}$ and $\mathfrak{t}$ by $\mathfrak{t}^{*}$, then $\mathfrak{h}=\mathfrak{h}^{*}$ and $\mathrm{t}=\mathrm{t}^{*}$.

Proof. Sufficiency is an immediate consequence of Theorem (3.4). Necessity requires some additional observations. Suppose then $\varphi$ is an isomorphism from $\mathfrak{R}_{T}^{*}(X, Y, \mathfrak{f})$ onto $\mathfrak{R}_{T}^{*}(U, V, \mathfrak{g})$. The existence of the bijections $\mathfrak{h}$ and $t$ (as well as their uniqueness) is a consequence of Theorem (3.4). It remains for us to prove that they are, in fact, $\Re$-bijections. We do so first for $\mathfrak{h}$. Let $H \in \mathscr{F}_{X}$. Then by (3.1.3), $\mathfrak{f}^{+}[H] \in \mathscr{F}_{\boldsymbol{Y}}$ and there exists an $\mathscr{F}_{\boldsymbol{Y}}$-invariant function $g$ in $\mathfrak{R}_{\boldsymbol{T}}^{*}(U, V, \mathfrak{g})$ such that $g[Y]=\mathfrak{f}^{\leftarrow}[H]$. Let $h$ be the $\Re$-function from $X$ onto $Y$ whose existence is guaranteed by (3.1.1) and set $f=g \circ h$. Note that $f[X]=\mathfrak{f}^{-}[H]$ and hence $(\mathfrak{f} \circ f)[X]=H$. From this and the diagram, we get

$$
\begin{aligned}
\mathfrak{h}[H] & =\mathfrak{h}[(\mathfrak{f} \circ f)[X]]=(\mathfrak{h} \circ \mathfrak{f} \circ f)[X]=(\mathfrak{g} \circ \mathfrak{t} \circ f)[X] \\
& =(\mathfrak{g} \circ \varphi(f) \circ \mathfrak{h})[X]=(\mathfrak{g} \circ \varphi(f))[U] .
\end{aligned}
$$

Since both $\mathfrak{g}$ and $\varphi(f)$ are $\Re$-functions, $\mathfrak{g} \circ \varphi(f)$ is also and hence $(\mathfrak{g} \circ \varphi(f))[U] \in \mathscr{F}_{U}$. In a similar manner, one shows $\mathfrak{h}^{-}$is an $\Re$-function and it follows that $\mathfrak{h}$ is an $\Re$-bijection.

Now suppose $H \in \mathscr{F}_{Y}$. Then there exists an $\Re$-function $g$ from $Y$ onto $H$ and an $\Re$-function $h$ from $X$ onto $Y$. Let $f=g \circ h$. Then $f[X]=H$ and, from the diagram, we get

$$
\mathfrak{t}[H]=(\mathfrak{t} \circ f)[X]=(\varphi(f) \circ \mathfrak{h})[X]=\varphi(f)[U] .
$$

But $\varphi(f)[U] \in \mathscr{F}_{\boldsymbol{V}}$. The fact that $\mathrm{t}^{+}$is an $\Re$-function follows in the same manner and we conclude $t$ is also an $\Re$-bijection.

This latter result, together with some results of Section 2, generalizes several theorems in [7] on semigroups of closed functions and semigroups of connected functions. Let $X$ and $Y$ be $T_{1}$ topological spaces and let $\mathfrak{j}$ be a closed continuous mapping from $Y$ onto $X$. Let $p$ and $q$ be distinct points of $X$. Since card $(X) \leqq$ card $(Y)$, there exist two distinct points $y$ and $z$ of $Y$. Define a function $f$ from $X$ into $Y$ by $f(p)=y$ and $f(x)=z$ for $x \neq p$. Then $f$ is a closed mapping. Suppose, in addition, there exists a closed mapping from $X$ onto $Y$. Then the triple $(X, Y, \mathfrak{f})$ is $\Re$-admissible where the $\Re$-families are the families of all closed subsets of $X$ and $Y$ respectively. We recall from Corollary (2.6) that these are indeed $\Re$-families. In this case $\mathfrak{R}_{T}^{*}(X, Y, \mathfrak{f})$ is the semigroup of all closed functions mapping $X$ into $Y$ where the product of $f$ and $g$ is defined to be $f \circ f \circ g$. This particular $\Re_{T}^{*}$-semigroup will be denoted by $\mathcal{S} \mathfrak{I}_{T}^{*}(X, Y, \mathfrak{i})$ and will be referred to as a (CI $I_{T^{*}}^{*}$-semigroup. With this convention, the following corollary is an immediate consequence of Theorem (3.5). 
Corollary (3.6). A bijection $p$ from $\mathfrak{C I}_{T}^{*}(X, Y, \mathfrak{f})$ onto $\mathfrak{C I}_{T}^{*}(U, V, \mathfrak{g})$ is an isomorphism if and only if there exists a unique homeomorphism hy from $X$ onto $U$ and a unique homeomorphism $\mathrm{t}$ from $Y$ onto $V$ such that for each $f$ in $\widehat{B} \mathfrak{l}_{T}^{*}(X, Y, \mathfrak{f})$, the following diagram commutes.

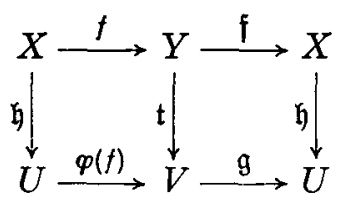

In [7] we used the symbol $\Gamma(X)$ to denote the semigroup of all closed functions mapping the $T_{1}$ topological space $X$ into itself where the binary operation is composition. By letting $X=Y, U=V$ and $f$ and $g$ be the identity functions in Corollary (3.6), we obtain

Corollary (3.7). Let $X$ and $U$ be $T_{1}$ spaces. A mapping $\varphi$ from $\Gamma(X)$ onto $\Gamma(U)$ is an isomorphism if and only if there exists a homeomorphism $\mathfrak{h}$ from $X$ onto $U$ such that $\varphi(f)=\mathfrak{h} \circ f \circ \mathfrak{h}^{+}$for each $f$ in $\Gamma(X)$.

This latter result is Theorem (2.10) of [7].

Let us recall that Theorem (2.8) of this paper states that the family of all connected subsets of a connected completely regular Hausdorff space with cardinality $c$ is an $\Re$-family. The techniques used in proving Theorem (2.8) also yield

Lemma (3.8). Let $X$ and $Y$ be connected completely regular Hausdorff spaces with cardinality equal to $c$. Furthermore, let $\mathfrak{f}$ be a connected function (i.e., a function which takes connected sets into connected sets) from $Y$ onto $X$ with the property that preimages of connected sets are connected. Then the triple $(X, Y, \mathfrak{f})$ is $\Re$-admissible where the $\Re$-families are the connected subsets of $X$ and $Y$ respectively.

Proof. Let $p$ and $q$ be distinct points of $X$. Since $X$ is completely regular and Hausdorff there exists a continuous function $f$ mapping $X$ into the closed unit interval $I$ such that $f(p)=0$ and $f(q)=1$. Since $X$ is connected, $f[X]=I$. Let $g$ be the function mapping $I$ onto $I$ which is defined in the proof of Theorem (2.8). Since $Y$ has cardinality $c$, there exists a bijection $k$ mapping $I$ onto $Y$. Then $(k \circ g \circ f)(p) \neq(k \circ g \circ f)(q)$ and for any connected subset $H$ of $X, f[H]$ is either a single point of $I$ or a nondegenerate subinterval of $I$. In the former case $(k \circ g \circ f)[H]$ is a single point while in the latter case $(k \circ g \circ f)[H]=Y$. Thus $k \circ g \circ f$ is a connected function mapping $X$ onto $Y$. Therefore, both conditions (3.1.1) and (3.1.2) are satisfied and the triple $(X, Y, \mathfrak{f})$ is $\Re$-admissible.

The $\mathfrak{R}_{T}^{*}$-semigroup associated with this particular triple is simply the 
semigroup of all connected functions mapping $X$ into $Y$ where the product $f g$ of two such functions is defined by $f g=f \circ f \circ g$. This semigroup will be denoted by $\mathbb{C} t_{r}^{*}(X, Y, \mathfrak{f})$ and will be referred to as a $\mathfrak{C} \mathrm{t}_{T}^{*}$-semigroup.

Pervin and Levine in [10] refer to a bijection $\mathfrak{h}$ with the property that both $\mathfrak{h}$ and $\mathfrak{h}^{+}$are connected mappings as a biconnected mapping. We use this terminology in stating the following corollary which is a consequence of Theorem (3.5) and Lemma (3.8).

Corollary (3.9). Let $X, Y, U$ and $V$ be connected completely regular Hausdorff spaces with cardinality equal to $c$. Let $\mathrm{f}$ be a connected function from $Y$ onto $X$ with the property that preimages of connected sets are connected and, similarly, let $g$ be a connected function from $V$ onto $U$ with the property that preimages of connected sets are connected. Then a bijection $\varphi$ from $\mathrm{Ct}_{T}^{*}(X, Y, \mathfrak{f})$ onto $\mathrm{Ct}_{T}^{*}(U, V, \mathfrak{g})$ is an isomorphism if and only if there exist unique biconnected mappings $\mathfrak{h}$ and $\mathrm{t}$ from $X$ onto $U$ and $Y$ onco $V$ respectively such that for any $f$ in $\mathrm{Ct}_{T}^{*}(X, Y, \mathfrak{i})$, the following diagram commutes.

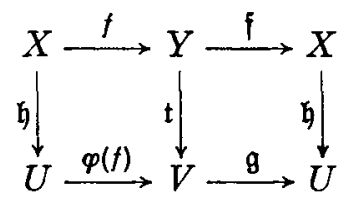

Theorem (3.10) of [10] states that if $X$ and $Y$ are locally connected compact Hausdorff spaces, then any biconnected mapping from $X$ onto $Y$ is a homeomorphism. This fact, together with Corollary (3.9) of this paper results in

Corollary (3.10). Let $X, Y, U$ and $V$ be compact connected locally connected Hausdorff spaces with cardinality equal to $c$. Let the functions $f$ and $\mathrm{g}$ satisfy the same conditions as in the statement of the previous corollary. Then a bijection $\varphi$ from $\mathfrak{C t}_{T}^{*}(X, Y, \mathfrak{f})$ onto $\mathfrak{C t}_{T}^{*}(U, V, \mathfrak{g})$ is an isomorphism if and only if there exist unique homeomorphisms $h$ and $\mathfrak{t}$ from $X$ onto $U$ and $Y$ onto $V$ respectively such that for each $f$ in ${ } \mathrm{t}_{T}^{*}(X, Y, \mathfrak{f})$, the following diagram commutes.

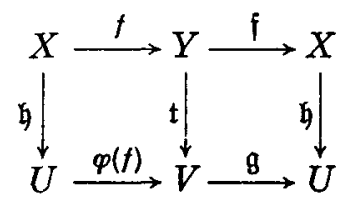

In [7], the semigroup of all connected functions mapping $X$ into $X$ under the binary operation of composition was denoted by $T(X)$. If we let $X=Y, U=V$ and both $f$ and $g$ be identity mappings in Corollary (3.10), we get 
Corollary (3.11). Let $X$ and $U$ be compact connected locally connected Hausdorff spaces with cardinality equal to $c$. Then a bijection $\varphi$ from $T(X)$ onto $T(U)$ is an isomorphism if and only if there exists a homeomorphism $\mathfrak{h}$ from $X$ onto $U$ such that $\varphi(f)=\mathfrak{h} \circ f \circ \mathfrak{h}^{+}$for each $f$ in $T(X)$.

This latter result is also a consequence of Theorems (3.2) and (3.5) of [7].

\section{Automorphisms of $\mathfrak{R}_{T}^{*}$-semigroups}

Let $\mathscr{S}$ be an arbitrary semigroup. We refer to an automorphism $\varphi$ of $\mathscr{S}$ as an inner automorphism if there exist elements $a$ and $b$ of $\mathscr{S}$ such that $\varphi(x)=a x b$ for each $x$ in $\mathscr{S}$. Our first result in this section gives several conditions which are equivalent to the condition that there exists an inner automorphism of $\mathfrak{R}_{T}^{*}(X, Y, \mathfrak{f})$ when the triple $(X, Y, \mathfrak{f})$ is $\Re$-admissible. Before stating this theorem, we introduce an additional bit of notation. If $X$ is any $\Re$-space and $\mathfrak{i}$ is the identity mapping on $X$, then the triple $(X, X, \mathfrak{i})$ is $\Re$-admissible and the binary operation of the $\Re_{T^{*}}^{*}$-semigroup $\mathfrak{R}_{T}^{*}(X, X, \mathfrak{i})$ is ordinary composition, i.e., $t g=f \circ g$ for all $f, g$ in $\Re_{T}^{*}(X, X, \mathfrak{i})$. We will denote this particular semigroup by $\Re_{T}^{*}(X)$.

TheOREM (4.1). Let $(X, Y, \mathfrak{f})$ be an $\Re$-admissible triple. Then the following statements concerning the $\mathfrak{R}_{T}^{*}$-semigroup $\mathfrak{R}_{T}^{*}(X, Y, \mathfrak{f})$ are equivalent.

(4.1.1) $\Re_{T}^{*}(X, Y, f)$ has an identity.

(4.1.2) $\Re_{T}^{*}(X, Y, \mathfrak{f})$ has a left identity.

(4.1.3) $\mathfrak{f}$ is an $\Re$-bijection from $Y$ onto $X$.

(4.1.4) $\mathfrak{R}_{T}^{*}(X, Y, \mathfrak{f})$ is isomorphic to $\mathfrak{R}_{T}^{*}(X)$.

(4.1.5) $\Re_{T}^{*}(X, Y, \mathfrak{f})$ is isomorphic to $\Re_{T}^{*}(Y)$.

(4.1.6) Every automorphism of $\Re_{T}^{*}(X, Y, \mathfrak{f})$ is inner.

(4.1.7) At least one automorphism of $\mathfrak{R}_{T}^{*}(X, Y, \mathfrak{f})$ is inner.

Proof. It is evident that (4.1.1) $\Rightarrow(4.1 .2)$. We begin by proving

(4.1.2) $\Rightarrow$ (4.1.3). Let $\mathfrak{l}$ be a left identity of $\Re_{T}^{*}(X, Y, \mathfrak{f})$ and for $y$ in $Y$, let $\boldsymbol{y}$ denote the constant function in $\Re_{T}^{*}(X, Y, \mathfrak{f})$ which is defined by $\boldsymbol{y}(\boldsymbol{x})=\boldsymbol{y}$ for each $x$ in $X$. Then $y=\boldsymbol{y}(\mathfrak{f}(y))=\mathfrak{l} y(\mathfrak{f}(y))=\mathfrak{l} \circ \mathfrak{f} \circ \boldsymbol{y}(\mathfrak{f}(y))=\mathfrak{l}(\mathfrak{f}(y))=\mathfrak{l} \circ \mathfrak{f}(y)$. Thus $\mathfrak{l} \circ f$ is the identity mapping on $Y$ which implies $f$ is an injection and thus a bijection onto $X$. Moreover, $\mathfrak{f}$ is an $\Re$-bijection since, by assumption, $(X, Y, \mathfrak{f})$ is $\Re$-admissible.

$(4.1 .3) \Rightarrow(4.1 .4)$. Since $f$ is a bijection onto $X$, the mapping $\varphi$ from $\Re_{T}^{*}(X, Y, f)$ onto $\Re_{T}^{*}(X)$ defined by $\varphi(f)=f \circ f$ is also a bijection. Moreover, for any $f$ and $g$ in $\Re_{T}^{*}(X, Y, \mathfrak{f}), \varphi(f g)=\varphi(f \circ f \circ g)=f \circ f \circ f \circ g=\varphi(f) \circ \varphi(g)$. 
Thus $\varphi$ is an isomorphism from $\Re_{T}^{*}(X, Y, \mathfrak{f})$ onto $\Re_{T}^{*}(X)$.

(4.1.4) $\Rightarrow$ (4.1.5). Since $\Re_{T}^{*}(X)$ has an identity (namely, the identity mapping on $X), \Re_{T}^{*}(X, Y, \mathfrak{f})$ does also. Just as in the proof that (4.1.2) $\Rightarrow(4.1 .3)$, one shows that $f$ is an $\Re$-bijection. Because of this, the mapping from $\Re_{T}^{*}(X, Y, \mathfrak{f})$ onto $\mathfrak{R}_{T}^{*}(Y)$ defined by $\varphi(f)=f \circ \mathfrak{f}$ is a bijection. Furthermore, for any $f, g$ in $\Re_{T}^{*}(X, Y, \mathfrak{f}), \varphi(f g)=\varphi(f \circ \mathfrak{f} \circ g)=f \circ \mathfrak{f} \circ g \circ \mathfrak{f}=\varphi(f) \circ \varphi(g)$. Thus $\Re_{T}^{*}(X, Y, \mathfrak{f})$ and $\Re_{T}^{*}(Y)$ are isomorphic.

$(4.1 .5) \Rightarrow(4.1 .6)$. As in the previous case, since $\mathfrak{R}_{T}^{*}(Y)$ has an identity, $\mathfrak{R}_{T}^{*}(X, Y, \mathfrak{f})$ does also and this implies $\mathfrak{f}$ is an $\Re$-bijection. Now let $\varphi$ be an automorphism of $\Re_{T}^{*}(X, Y, \mathfrak{f})$. By Theorem (3.5), there exist unique $\Re$ bijections $\mathfrak{h}$ and $t$ from $X$ onto $X$ and $Y$ onto $Y$ respectively such that for any $f$ in $\Re_{T}^{*}(X, Y, \mathfrak{f})$ the following diagram commutes.

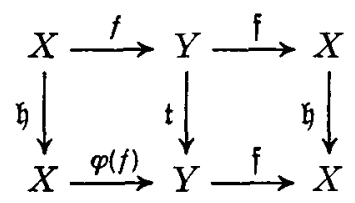

Thus, for each $f$ in $\mathfrak{R}_{T}^{*}(X, Y, \mathfrak{f}), \varphi(f)=\mathrm{t} \circ f \circ \mathfrak{h}^{*}$. From this, and the fact that $f$ is an $\Re$-bijection, we get

$$
\varphi(f)=\left(\mathfrak{t} \circ \mathfrak{f}^{+}\right) \circ \mathfrak{f} \circ f \circ \mathfrak{f} \circ\left(f^{+} \circ \mathfrak{h}^{+}\right)=\left(\mathfrak{t} \circ \mathfrak{f}^{+}\right) f\left(\mathfrak{f}^{+} \circ \mathfrak{h}^{+}\right)
$$

for each $f$ in $\mathfrak{R}_{T}^{*}(X, Y, \mathfrak{f})$. Since both $\mathrm{t} \circ \mathfrak{f}^{\leftarrow}$ and $\mathfrak{f}^{\leftarrow} \circ \mathfrak{h}^{\leftarrow}$ are elements of $\Re_{T}^{*}(X, Y, \mathfrak{f})$, it follows that $\varphi$ is an inner automorphism.

It is evident that $(4.1 .6) \Rightarrow(4.1 .7)$ so to complete the proof, we need only show that $(4.1 .7) \Rightarrow(4.1 .1)$. To do this we turn to a result of M. L. Vitanza [11]. She has shown that if $a$ and $b$ are any pair of elements of a semigroup $\mathscr{S}$ such that mapping $\varphi$ from $\mathscr{S}$ into $\mathscr{S}$, defined by $\varphi(x)=a x b$ for each $\boldsymbol{x}$ in $\mathscr{S}$, is an automorphism, then $\mathscr{S}$ has an identity and, moreover, $a$ and $b$ are inverses of each other relative to that identity. Hence, any semigroup which has an inner automorphism must have an identity.

Now suppose $X$ and $Y$ are $\Re$-spaces and $(X, Y, \mathfrak{f})$ is an $\Re$-admissible triple. Let $\mathscr{D}_{\mathfrak{f}}$ denote the family of all point inverses of $\mathfrak{f}$, i.e.,

$$
\mathfrak{D}_{\mathrm{f}}=\left\{\mathfrak{f}^{\leftarrow}\{x\}: x \in X\right\} .
$$

Then $\mathscr{D}_{\mathcal{f}}$ is a subfamily of $\mathscr{F}_{Y}$ (the $\Re$-family of subsets associated with $Y$ ) consisting of mutually disjoint nonempty subsets of $Y$. We let $G_{\mathfrak{R}}\left(\mathfrak{D}_{\mathrm{f}}\right)$ denote the group of all $\Re$-bijections $h$ from $Y$ onto $Y$ with the property that $h[A] \in \mathscr{D}_{\mathrm{f}}$ for each $A \in \mathscr{D}_{\mathrm{F}}$. Using the same technique as in the proof of Lemma (3.5) of [9], we prove 
Lemma (4.2). Let $\mathrm{t}$ be an $\Re$-bijection from $Y$ onto $Y$. Then there exists an $\Re$-bijection $\mathfrak{h}$ from $X$ onto $X$ such that $\mathfrak{h} \circ \mathfrak{f}=\mathfrak{f} \circ \mathfrak{t}$ if and only if $\mathfrak{t} \in G_{\mathfrak{r}}\left(\mathfrak{D}_{\mathfrak{f}}\right)$.

Proof. We use Lemma (3.2) of [8] which we state as follows:

(4.2.1). Suppose $f$ maps $Y$ onto $X, \mathfrak{g}$ maps $Y$ onto $Z$ and $t$ is a bijection from $Y$ onto $Y$. Then there exists a bijection $\mathfrak{h}$ from $X$ onto $Z$ such that $\mathfrak{h} \circ \mathfrak{f}=\mathfrak{g} \circ \mathfrak{t}$ if and only if $\mathrm{t}[A] \in \mathscr{D}_{\mathfrak{g}}$ for each $A$ in $\mathscr{D}_{\mathfrak{f}}$.

First suppose $t \in G_{\mathfrak{r}}\left(\mathfrak{D}_{\mathfrak{F}}\right)$. By (4.2.1), there exists a bijection $\mathfrak{h}$ from $X$ onto $X$ such that $\mathfrak{h} \circ \mathfrak{f}=\mathfrak{f} \circ \mathfrak{t}$. Now let $H \in \mathscr{F}_{X}$. Since $(X, Y, \mathfrak{f})$ is admissible, $\mathfrak{f}^{-}[H] \in \mathscr{F}_{Y}$ and thus $\mathrm{t}\left[\mathfrak{f}^{-}[H]\right] \in \mathscr{F}_{Y}$. But this implies $\mathfrak{h}[H]=\mathfrak{f}\left[\mathrm{t}\left[\mathfrak{f}^{-}[H]\right]\right] \in \mathscr{F}_{X}$. Hence $\mathfrak{h}$ is an $\Re$-function from $X$ onto $X$. One shows, in a similar manner, that $\mathfrak{h}^{-}$is also an $\mathfrak{R}$-function and it follows that $\mathfrak{h}$ is an $\mathfrak{R}$-bijection. The necessity portion of the proof is an immediate consequence of (4.2.1).

With this latter result, we are now ready to determine the automorphism groups of $\Re_{T}^{*}$-semigroups when the triples involved are admissible.

Theorem (4.3). Let $X$ and $Y$ be $\Re$-spaces and suppose the triple $(X, Y, \mathfrak{f})$ is $\Re$-admissible. Then the automorphism group of $\Re_{T}^{*}(X, Y, \mathfrak{f})$ is isomorphic to $G_{\mathfrak{r}}\left(\mathcal{D}_{\mathfrak{f}}\right)$.

Proof. Let $\mathfrak{A}$ denote the automorphism group of $\mathfrak{R}_{T}^{*}(X, Y, \mathfrak{f})$ and define a mapping $\Phi$ from $\mathfrak{A}$ into $G_{\mathfrak{q}}\left(\mathscr{D}_{\mathfrak{q}}\right)$ as follows: let $\varphi$ be an element of $\mathfrak{A}$. Then according to Theorem (3.5), there exist unique $\mathfrak{R}$-bijections $\mathfrak{h}$ and $\mathbf{t}$ from $X$ onto $X$ and $Y$ onto $Y$ respectively such that for each $f$ in $\Re_{T}^{*}(X, Y, \mathfrak{f})$, the following diagram is commutative.

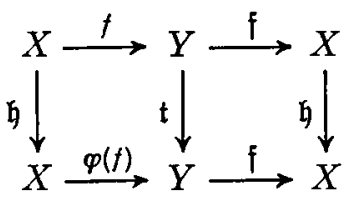

Define $\Phi(\varphi)=\mathrm{t}$. Since $\mathfrak{h} \circ \mathfrak{f}=\mathfrak{f} \circ \mathrm{t}$, it follows from Lemma (4.2) that $t \in G_{\mathfrak{P}}\left(\mathscr{D}_{\mathfrak{f}}\right)$ and hence, $\Phi$ is indeed a mapping from $\mathfrak{A}$ into $G_{\mathfrak{P}}\left(\mathscr{D}_{\mathfrak{f}}\right)$. One shows in a routine manner that $\Phi$ is a homomorphism. Now let $t$ be an arbitrary element of $G_{\mathfrak{R}}\left(D_{\mathfrak{F}}\right)$. Then by Lemma (4.2), there exists an $\Re$ bijection $\mathfrak{h}$ mapping $X$ onto $X$ such that $\mathfrak{h} \circ \mathfrak{f}=\mathfrak{f} \circ \mathfrak{t}$. It follows from Theorem (3.5) that the mapping $\varphi$ from $\mathfrak{R}_{T}^{*}(X, Y, \mathfrak{f})$ onto itself, defined by $\varphi(f)=\mathrm{t} \circ f \circ \mathfrak{h}^{-}$, is an automorphism. Since $\Phi(\varphi)=\mathrm{t}$, it follows that $\Phi$ is an epimorphism from $\mathfrak{A}$ onto $G_{\mathfrak{R}}\left(\mathfrak{D}_{\mathfrak{f}}\right)$. Now suppose $\varphi$ is in the kernel of $\Phi$. Again we use Theorem (3.5) to conclude the existence of unique bijections $\mathfrak{h}$ and $\mathfrak{t}$ such that the previous diagram commutes. But since $\varphi$ is in the kernel of $\Phi$ and $\Phi(\varphi)=\mathrm{t}$, it follows that $\mathrm{t}$ is the identity mapping on $Y$. Let $x$ be any point of $X$. Then there exists a point $y$ in $Y$ such that 
$\mathfrak{f}(y)=x$. From the diagram, we see that $\mathfrak{h}(x)=\mathfrak{h}(f(y))=\mathfrak{f}(\mathfrak{t}(y))=\mathfrak{f}(y)=x$. Thus, $\mathfrak{h}$ is the identity mapping on $X$ and it follows that $\varphi$ is the identity mapping on $\Re_{T}^{*}(X, Y, \mathfrak{f})$, i.e., $\varphi$ is the identity of $\mathfrak{A}$. This proves $\Phi$ is an isomorphism from $\mathfrak{A}$ onto $G_{\mathfrak{P}}\left(\mathfrak{D}_{\mathfrak{F}}\right)$.

Now let $X$ be a $T_{1}$ topological space and let $\mathscr{F}_{X}$ denote the family of all closed subsets of $X$. Then $\mathscr{F}_{X}$ is an $\Re$-family and the triple $(X, X, \mathfrak{i})$ is $\Re$-admissible where $i$ denotes the identity mapping on $X$. In this particular case, $G_{\mathfrak{R}}\left(\mathscr{D}_{\mathfrak{f}}\right)$ is just the group (under composition) of all homeomorphisms on $X$. Recalling that $\Gamma(X)$ denotes the semigroup, under composition, of all functions mapping $X$ into $X$, we apply Theorem (4.3) to obtain

CoROLlary (4.4). If $X$ is a $T_{1}$ topological space, then the automorphism group of $\Gamma(X)$ is isomorphic to the group, under composition, of all homeomorphisms on $X$.

Using the latter corollary and a very intersting result due to J. de Groot, we are able to prove

COROLLARY (4.5). Every group is isomorphic to the automorphism group of some semigroup.

Proof. Let $G$ denote an arbitrary group. Theorem 8 , page 98 of [3] states that there exists a compact connected Hausdorff space $X$ such that $G$ is isomorphic to the group of all homeomorphisms on $X$. But by Corollary (4.4), this group of homeomorphisms is isomorphic to the automorphism group of $\Gamma(X)$, the semigroup of all closed functions on $X$. Hence $G$ is isomorphic to the automorphism group of $\Gamma(X)$.

\section{References}

[1] G. Birkhoff, 'Sobre los grupos de automorfismos', Rev. Union mat. Argent. 11 (1945), $155-157$.

[2] A. H. Clifford and G. B. Preston, The algebraic theory of semigroups, Mathematical Surveys, Number 7 (Amer. Math. Soc., 1961).

[3] J. de Groot, 'Groups represented by homeomorphism groups, I', Math. Annalen 138 (1959), 80-102.

[4] J. L. Kelley, General Topology (Van Nostrand, Princeton, 1955).

[5] C. Kuratowski, Topologie II (Warsaw, 1950).

[6] E. S. Ljapin, Semigroups, Vol. 3, Translations of Mathematical Monographs (Amer. Math. Soc., 1963).

[7] K. D. Magill, Jr., 'Semigroups of functions on topological spaces', Proc. London Math. Soc. (3) 16 (1966), $507-18$.

[8] K. D. Magill, Jr., 'Semigroup structures for families of functions, I; Some homomorphism theorems', Journ. A ustralian Math. Soc. 7 (1967), 81-94.

[9] K. D. Magill, Jr., 'Semigroup structures for families of functions, II; Continuous functions', Journ. Australian Math. Soc. 7 (1967), 95-107.

[10] W. J. Pervin and N. Levine, 'Connected mappings of Hausdorff spaces', Proc. Amer. Math. Soc., 9 (1958), $488-96$.

[11] M. L. Vitanza, 'Mappings of semigroups associated with ordered pairs', Amer. Math. Monthly 73 (1966), 1078-1082. 